\title{
THE INFLUENCE OF WETTABILITY ON THE PETROPHYSICAL PARAMETERS OF RESERVOIR ROCKS**
}

\section{INTRODUCTION}

Wettability is one of the most important parameters in reservoir engineering. It controls fluid location, flow and distribution in a porous medium. In reservoir rock where different liquids are present, it is very important to obtain information about the type of wettability of the rock. This is because the understanding of the relationship between wettability and distribution of water and oil in the pore space is necessary to assess the efficiency of oil recovery. As a consequence, wettability affects all of the petrophysical properties of the reservoir $[3,6]$.

Wettability refers to the tendency of one fluid to spread on or adhere to a solid surface in the presence of immiscible fluids. In natural porous media, the wettability varies from point to point depending on surface roughness, immobile adsorbed liquid layers, and the adsorptive properties of the mineral constituents [13]. Anderson reported that coal, graphite, sulfur, talc, talc-like silicates, and many sulfides are probably neutrally wet to oil-wet [5]. On the other hand, most common minerals such as quartz, carbonates, and sulfates are strongly water wet [13].

The wettability of the reservoir rock controls the distribution of oil and water and affects their movement through pore spaces. Understanding wettability in porous media is a difficult problem and controlling it to modify the behavior of reservoir rock presents a more complex one. Numerous methodologies for studying, measuring, and altering the wettability of reservoir rocks are found in literature [1,3-10, 13, 16, 18, 20, 21, 25, 28]. No satisfactory method exists for in situ measurement of wettability, and therefore it is necessary to estimate the wettability of reservoir rocks from laboratory measurements.

The article presents the petrophysical parameter characteristic of rock dependent wettability and the analysis of various numerous experimental work done by many researchers was conducted.

* AGH University of Science and Technology, Faculty of Drilling, Oil and Gas, Krakow, Poland

** Work performed within the statutory research program of AGH UST 11.11.190.555 


\section{INFLUENCE OF WETTABILITY ON CAPILLARY PRESSURE}

Capillary pressure is an important reservoir property because it directly or indirectly affects other properties such as residual saturations and relative permeability curves. The relationship between capillary pressure and phase saturation is a function of wettability, pore structure, interfacial tension, rock properties, and saturation history or hysteresis $[4,8]$. When two immiscible fluids are in contact in the interstices of a porous medium, a discontinuity in pressure exists across the interface separating them. The difference in pressure $(P c)$ is called capillary pressure. The capillary pressure is dependent on the interfacial tension, pore size, and wetting angle. Capillary pressure is the most fundamental rock/fluid property in multiphase flow, just as porosity and permeability are for single phase flow in oil and gas reservoirs. Capillary pressure curves directly determine irreducible water saturation, residual oil saturation, and rock wettability and can be used to determine the water oil contact point and approximate oil recovery. Water flood performance is also significantly affected by the capillary pressure of the rock [13].

Capillary pressure can be defined as the pressure difference between the non-wetting phase pressure and wetting phase pressure. It is mathematically expressed as [4]:

$$
P_{c}=P_{n w}-P_{w}=\frac{2 \sigma \cos \theta}{r}=\left(\rho_{w}-\rho_{n w}\right) g h
$$

where:

$$
\begin{aligned}
P_{c} & - \text { capillary pressure }[\mathrm{Pa}], \\
P_{n w} & - \text { pressure of the non-wetting phase }[\mathrm{Pa}], \\
P_{w} & - \text { pressure of the wetting phase }[\mathrm{Pa}], \\
\sigma & - \text { surface tension }[\mathrm{N} / \mathrm{m}], \\
\theta & - \text { contact angle }\left[^{\circ}\right], \\
r & - \text { the radius of capillary tube }[\mathrm{m}], \\
\rho_{w}, \rho_{n w} & - \text { the wetting and non-wetting phase densities }\left[\mathrm{kg} / \mathrm{m}^{3}\right], \\
h & - \text { the height of the column of water in the capillary tube }[\mathrm{m}] .
\end{aligned}
$$

Capillary pressure data are not only important for obtaining reservoir rock properties such as pore size distribution, permeability, and water saturation profile within the oil reservoir but also provide important information for water flooding designs and reservoir simulation studies. This pressure is directly related to the wetting characteristics of the porous medium. Wettability influences capillary pressure saturation relationships as it controls the distribution of fluid in the pore space [1-3, 8, 10, 13, 14, 19, 27, 31, 33].

In a uniformly wetted porous medium, pore geometry effects and the extremely rough surface of the porous medium make the capillary pressure curve insensitive to wettability for small contact angles (less than about $50^{\circ}$ for drainage capillary pressure curves and less than about $20^{\circ}$ for spontaneous-imbibition capillary pressure curves). When the porous medium has fractional or mixed wettability, both the amount and distribution of the oil-wet and water-wet surfaces are important in determining the capillary pressure curve, residual saturation, and imbibition behavior. Imbibition also depends 
on the interaction of wettability, pore structure, initial saturation, and saturation history. Because of these interactions, there is a large range of contact angles where neither oil nor water will imbibe freely into a uniform wetted reservoir core. In contrast, it is sometimes possible for both fluids to imbibe freely into a core with fractional or mixed wettability $[2,8]$.

Graue et al. [14] studied the impact of wettability on capillary pressure. They used nine core plugs with different wettability indexes. The standard centrifuge technique was used to obtain the capillary pressure data. They observed that the drainage capillary pressure for the less water-wet core plugs showed lower drainage threshold pressure, sharper curvature for the drainage curve and higher irreducible water saturation than the strongly water-wet core plugs. During forced water invasion, the capillary pressure curve with less water-wet conditions become flatter than the water-wet condition cores. Residual oil saturation values were smaller for less water-wet cores [3].

Also Al-Garni [2] investigated the effect of wettability on capillary pressure (the drainage and imbibitions curves), and irreducible saturation for Saudi crude oils, using the rock centrifuge method. Due to the difference in oil compositions of the used crude oils (light, medium and heavy), there are marked changes in the capillary curves of the drainage cycles for the tested samples. Similarly, these marked changes between the capillary curves during the ambition cycles were occurred. The relationship between the capillary pressure, water saturation, and water saturation two stages should be investigated. Light, medium and heavy crude oils were used in samples A, B, and C respectively. The sample $\mathrm{C}$ (heavy crude oil) had the highest irreducible water saturation among the other two samples whereas the drainage cycle means increasing the wetting-phase saturation from its maximum to the irreducible minimum by increasing the capillary pressure from zero to a large positive value, therefore, used fluid (heavy) in sample C change the wettability of this sample towards oil-wet. Heavy oil has higher asphaltene concentrations than light oil. Asphaltene or polar components present in crude oil can adsorb on mineral surfaces and alter their wetting properties.

The difference in capillary pressure for various degrees of water-wetness is shown in Figure 1. Figure 1a shows the effect of wettability from moderately water-wet to strongly water-wet on the drainage capillary pressure curve is negligible. This effect is not negligible, however, for the imbibition curve (Fig. 1b). The importance of this observation is based on the rock wettability, the fluid displacement process and oil entrapment mechanism. During drainage, the nonwetting phase displacing the wetting phase and there is no trapped oil saturation. For the cases with uniform moderate to strong wettability, the rock is in contact with the wetting phase and the nonwetting phase flows through the center of the pore. This fluid displacement process causes little changes capillary pressure curve for this range of wettability. On the other hand, during the imbibition process the wetting phase displaces the nonwetting phase and the nonwetting phase is trapped. During this displacement process, the nonwetting phase was in contact with the pore walls in the absence of the wetting phase, and is removed and replaced with the wetting phase. This phase replacement and nonwetting phase entrapment occurs at different rates for different wettabilities and has a significant effect on capillary pressure $[4,27]$. 
a)

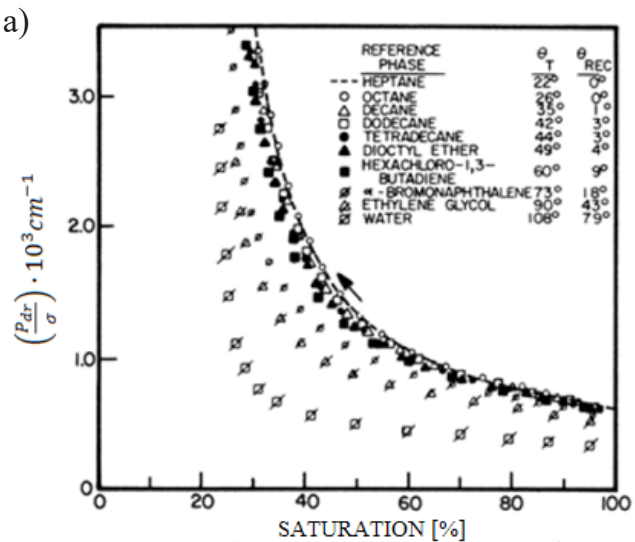

b)

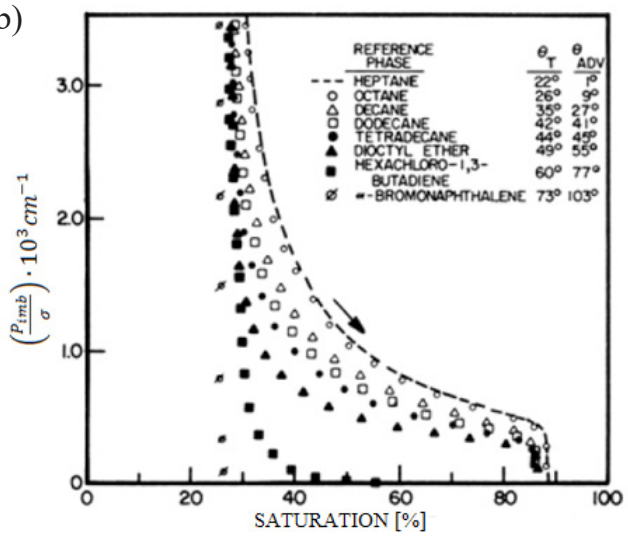

Fig. 1. Effect of wettability on water-wet capillary pressure curves [27]: a) drainage; b) imbibition

Killins et al. [19] studied the influence of strongly wetted systems on the imbibitions and drainage curves of capillary pressure for consolidated sandstones using the porous plate technique. Strongly water-wet systems have very different areas under the drainage and imbibitions curves. This is due to contact angle hysteresis (different contact angles depending on the direction of flow) and different displacement processes in drainage and imbibitions [3] (Fig. 2).
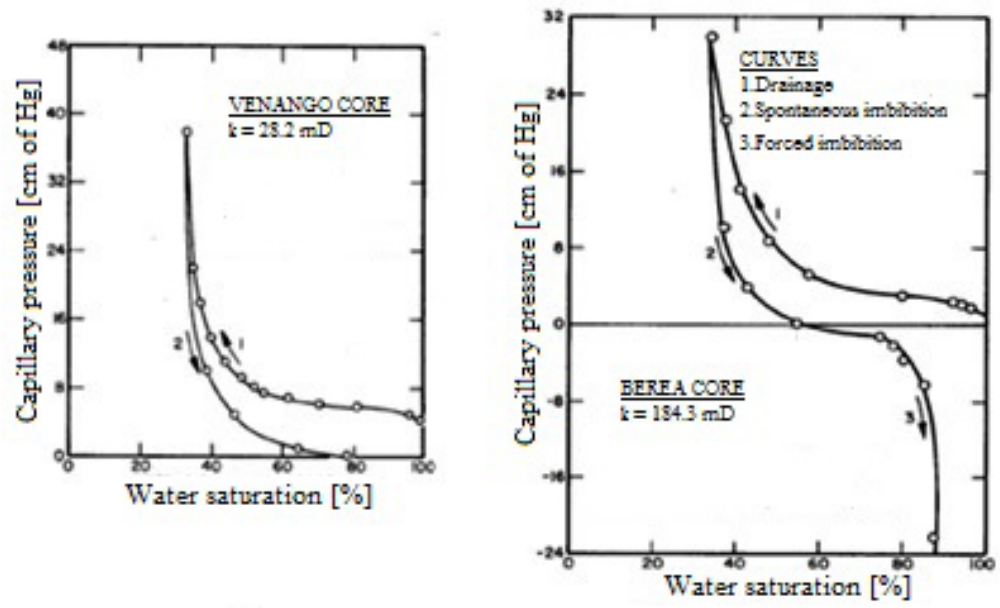

Fig. 2. Effect of wettability on water-wet capillary pressure curves [19, modified]

O'Carroll et al. [31] predicted two-phase capillary pressure/saturation relationships in fractional wettability systems. They presented a new two-phase capillary pressure/saturation model for application to the prediction of primary drainage and imbibition relations in fractional wettability media. That new model was based on an extension of Leverett scaling theory [3]. 


\section{INFLUENCE OF WETTABILITY ON RELATIVE PERMEABILITY}

Relative permeability characteristics are crucial in the displacement of hydrocarbons by water. It is a function of wettability, pore geometry, fluid distribution, saturation and saturation history. Wettability affects relative permeability by controlling the flow and spatial distribution of fluids in a porous media. The shapes of relative permeability curves are characteristic of the wetting qualities of the fluids (Fig. 3). Typically, as the system becomes more oil-wet, the water relative permeability increases and the oil relative permeability decreases. In the more oil-wet rock, the higher water saturation positioned in the center of the pores competing with the oil in the most permeable pathways, reducing the relative permeability to oil, and increasing the relative permeability to water [16].

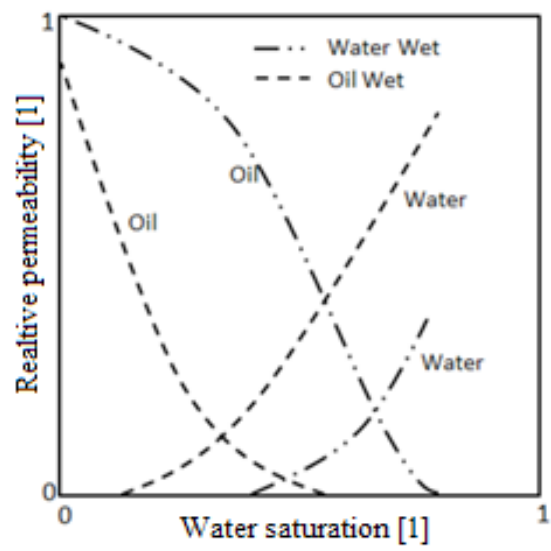

Fig. 3. Relative permeability of water-wet and oil-wet systems [16]

Wettability changes significantly affect the efficiency of waterflooding. During waterflood, strongly water-wet cores will tend to produce more oil at water breakthrough. While mixed-wet cores will continuously produce oil resulting in very low oil saturations $[3,20]$. The highest oil recovery will be obtained when flooding neutral-wet systems, while oil-wet systems will have the lowest recovery for only a few pore volumes injected $[3,15]$.

Research on the influence of wettability on relative permeability has been conducted by a number of authors $[2,3,9,10,13,14,15,17,32]$.

Owens and Archer [32] used Torpedo sandstones to conduct their experiments. The wettability of the sandstones was altered and the contact angles were measured by a photographic technique. The contact angle measured for the samples varied between $0-180^{\circ}$. The authors showed that water-oil relative permeability curves distinctly changed with the wettability change: at a given saturation, as water wettability decreases, the oil relative permeability decreases [3].

An example of the change in relative permeability with changes in wettability was obtained from Morrow [3, 4, 28]. The author measured steady state relative permeabilities with water and a refined oil, using powdered dolomite as the porous medium. Wettability was controlled with different concentrations of octanoic acid in the oil. Water advancing 
contact angles were measured on a smooth dolomite crystal. Wettability varied from waterwet $\left(\theta_{\text {adv }}=15^{\circ}\right)$, neutral-wet $\left(\theta_{\text {adv }}=100^{\circ}\right)$ to oil-wet $\left(\theta_{\text {adv }}=155^{\circ}\right)$ [3]. It is apparent from this example that the primary effects of changes in wettability are the increase in the water relative permeability curve and the decrease in the oil relative permeability curve when shifting from water-wet to oil-wet. This effect shifts the crossover point to lower water saturations. There is a decrease in the residual oil saturation from $28 \%$ to $20 \%$ and a corresponding increase in the water endpoint relative permeability as wettability changes to less water wet conditions [4].

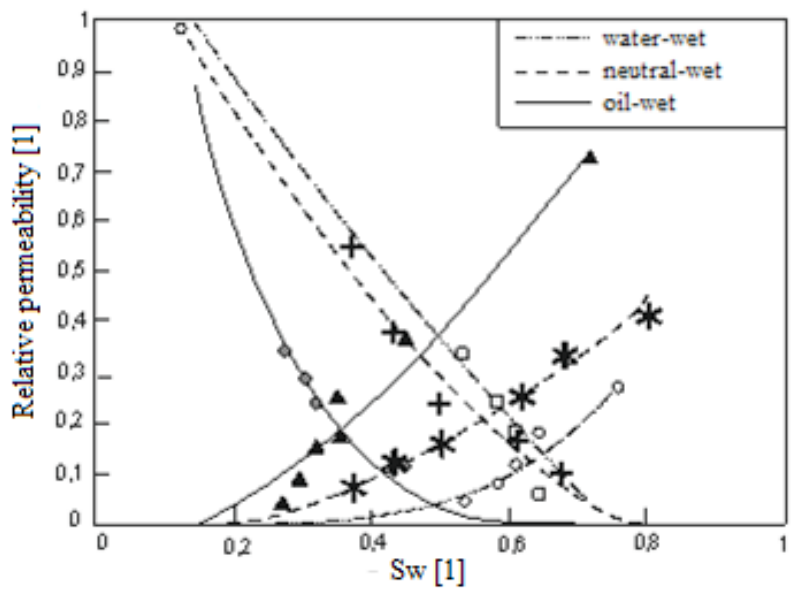

Fig. 4. The relative permeability of water and oil refined in a porous medium with different types of wettability [3]

Falode and Manuel [13] showed that the relative permeabilities of the wetting phase for an air-brine system are lower than therelative permeabilities of the wetting phase for an air-oilsystem, while it is the opposite for their nonwetting phase relative permeabilities. This shows that the wettability altering of the core samples from water-wet to oil-wet increases therelative permeability of the wetting phase. This shows that oil recovery can be improved using this method.

The three-phase flow in water-wet, oil-wet, and fractionally-wet sandpacks was studied by Hui and Blunt [17]. They use CT scanning to measure directly the oil and water relative permeabilities for three-phase gravity drainage. Researchers found that gas relative permeability is approximately twice in a water-wet system than in an oil-wet system at the same gas saturation. The water relative permeability in the water-wet sample and the oil relative permeability in the oil-wet sample are similar [13].

Morrow et al. [29] studied the effect of wettability changes on oil recovery. They observed that water relative permeability at residual oil saturation decreased when the system became less water-wet. Willhite [36] observed that oil relative permeability increases when the porous medium becomes oil-wet. The reasons for these contradictory results are still not very clear. It could be due to the heterogeneity of the cores or different methods used to measure relative permeability [3]. 


\section{INFLUENCE OF WETTABILITY ON ELECTRICAL RESISTIVITY}

Electrical resistivity is an important petrophysical property. Wettability and the saturation history of a core establishes the fluid distributions within the pore spaces and therefore controls the resistivity of the electrical pathways through the porous system. Resistivity is proportional to the impressed voltage and cross-sectional area and inversely proportional to the current flowing and the length of the electrical conductive path [12]:

$$
R=\left(\frac{E}{I}\right)\left(\frac{\phi A}{L}\right)
$$

where:

$$
\begin{aligned}
& R-\text { electrical resistivity }[\mathrm{ohm} \cdot \mathrm{m}], \\
& E-\text { impressed voltage [volt] }, \\
& I-\text { electric current [amper], } \\
& A-\text { cross-sectional area }\left[\mathrm{m}^{2}\right], \\
& L-\text { length of the electrical conductive path }[\mathrm{m}], \\
& \phi-\text { rock porosity }[1] .
\end{aligned}
$$

Rocks are generally classified as insulators, but inclusions of minerals such as some clays and metals can lower their resistivity and also hydrocarbons are treated as insulators. The resistivity of a solution containing dissolved salts (brine) placed in a reservoir with the same volumetric dimensions of a rock sample has a lower resistivity than the rock saturated with brine. The insulating rock grains diminish the cross-sectional area of the conducting brine, and the tortuosity of the porous pathways through the rock increases the distance that the current must traverse [10].

The variation in wettability will have an effect on Archie's saturation exponent $(n)$ and formation resistivity factor $\left(F_{r}\right)$, which are essential for determining the hydrocarbon saturation [7]. A number of laboratory studies have shown that the value of $n$ can vary whenaltering the wettability of the core. In order to understand how wettability affects the electrical response of a rock, $n$ must be measured at reservoir wetting conditions, else unreliable saturation values will be obtained [3].

Donaldson and Siddiqui [11] examined the effect of wettability on the saturation exponent and temperature. For both sandstones, the wettability increased linearly as wettability was changed from water-wet to strongly oil-wet (as indicated by the decrease of the USBM wettability index) at temperatures of $25^{\circ} \mathrm{C}$ and $78^{\circ} \mathrm{C}$ (Fig. 5). For both cases, the systems became more water-wet at the higher temperatures. Differences of the lines slopes may indicate differences in pore geometry between the two types of rocks. An increase in temperature also produces an increase in the electrical resistance of conductors [10].

The electrical resistivity at reservoir conditions on water-wet samples was measured by Mahmood et al. [24]. They found that the Archie saturation exponents decreased at increasing temperatures while the decrease became smaller at higher temperatures [34].

Keller showed that different values of resistivity can be obtained at the same rocks water saturation if the wettability is changed [18]. Values of the saturation exponent $n$ ranged from 1.5 to 11.7 for the same rock. Oil-wet rocks have a high resistivity because oil is an insulator. 
Even at very low water saturations, a water-wet sand sample will have a continuous water film along the sand grains surfaces from the entrance to the exit, which furnishes a conductive path for the electric current. In an oil-wet sand, oil is the continuous phase and contact with the pore walls. In this case water is the discontinuous phase, the electrical path is interrupted by the insulating oil. Consequently oil-wet sand resistivity is very high, and the Archie saturation exponent $\mathrm{n}$ is considerably greater than 2.0 [12].
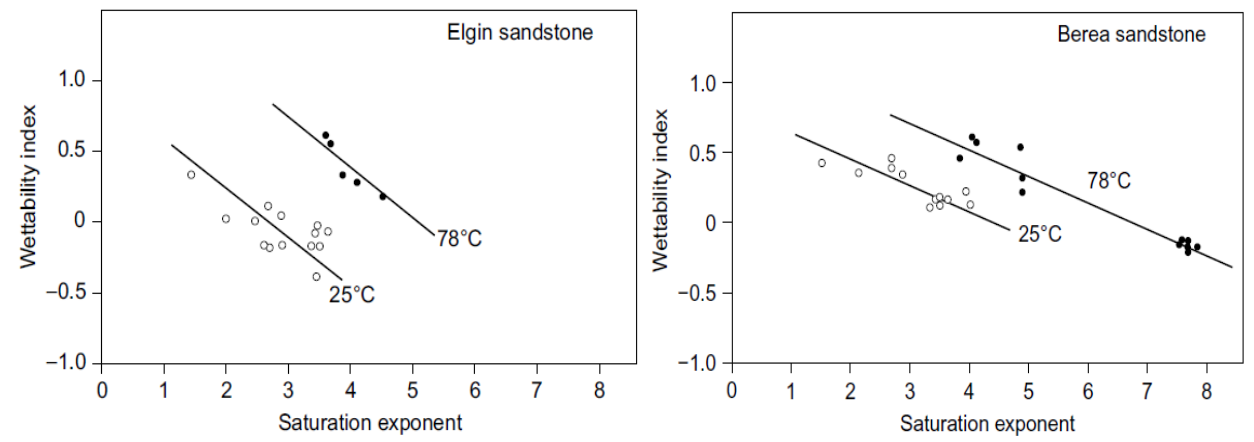

Fig. 5. Change in the Archie saturation exponent as a function of wettability and temperature for Berea and Elgin outcrop sandstone [12]

Sweeney and Jennings [35] measured the influence of wettability on the electrical response for different carbonate cores: water-wet, neutral-wet and oil-wet. The electrical resistivity of the cores was measured as a function of water saturation. The Archie saturation exponent was found to be 1.9, whereas, at oil-wet conditions $n$ was equal to 12 . This finding is consistent with Mungan and Moore's [30] results [3].

Determination of Archie saturation exponents at reservoir conditions were conducted by Longeron et al. [22]. They determined the resistivity indexes using refined oil, then the samples were restored and drained with crude oil. They found that the value of the Archie saturation exponents obtained using crude oil, were generally higher than the Archie saturation exponents obtained with refined oil. The increase of the Archie saturation exponents was attributed to the change in wetting properties. The Archie saturation exponents increased from 2.0 to 2.9 as the core became more oil-wet.

Morgan and Pirson [26] reported a very wide range of values for $n$, from 2.5 for strongly water-wet samples to 25.2 for strongly oil-wet packs of glass beads treated with progressively higher concentrations of silicone solutions [12].

Sondena et al. [33] compared capillary pressure measurements on intermediate and slightly water-wet samples, wettability preference at ambient conditions with samples of intermediate and slightly water-wet preference which changed to oil-wet (mixed-wet) preference during the capillary pressure measurement at reservoir conditions. The water saturated samples used at ambient conditions were displaced with refined oil and the twin samples used at reservoir conditions were displaced with live crude oil. From the results of the electrical resistivity measurements, the authors found that the resistivity indexes obtained at reservoir conditions were higher than the resistivity indexes obtained for the same water saturation at ambient conditions. The largest differences were found in the measurements 
at low water saturations where water was displaced by crude oil. The biggest increase in saturation exponent $n$ was 0.60 units with the highest saturation exponent at 2.30. The differences in resistivity indexes were related to a change in the wettability preference. Recently Longeron et al. [23] have observed a similar result. They also restored the samples during drainage with reservoir oil. The saturation exponent increased with decreasing water saturation [34].

\section{CONCLUSIONS}

The wettability of the reservoir rock is important because it is a major factor controlling the location, flow, and distribution of fluids in a reservoir. From the viewpoint of petrophysics, wettability influences different processes and parameters (water saturation calculation, core analysis experiments, residual oil saturation, water-flood behavior and simulated recovery processes). Wettability strongly affects the spatial distribution of conductive and nonconductive phases (brine and hydrocarbon, respectively) within the pore space and, therefore, the electric properties of the rock. These parameters affect the position of capillary pressure and the relative permeability curves which in turn affects the hydrocarbon flow in porous media.

Determination of wettability and its impact on the petrophysical parameters of reservoir rocks is still an interesting topic. Many researchers take on the determination of this petrophysical parameter. Knowledge of the wettability of a reservoir rock and its influence on petrophysical properties and the displacement of oil and gas are very important for determining of oil recovery mechanisms and recovery efficiency estimation. Although the literature has been showing a growing interest in the effect of wettability on fluid flow, in porous media itis still a subject of highly active investigation. Therefore, the present work focuses on gathering knowledge about reservoir wettability, which is key factor for many reasons ranging from oil migration from source rock to enhanced oil recovery.

Failure to take account wettability or the surface properties of the reservoir rock leads to undesirable effects during reservoir exploitation, such as sudden water cut, high value of residual oil in the reservoir, and, consequently, lower production of hydrocarbons and significant economic losses of oil companies.

\section{REFERENCES}

[1] Abdallah W.I., Carnegie A., Herold B., Edwards J., Forhdam E., Graue A., Habashy T., Seleznev N., Signer, C., Hussain, H., Montaron B., Ziauddin M.: Fundamentals of Wettability. Schlumberger Wettability Workshop, Bahrain, May 2007.

[2] Al-Garni M.T., Al-Anazi B.D.: Investigation of wettability effects on capillary pressure, and irreducible saturation for Saudi crude oils, using rock centrifuge. Oil and Gas Business, vol. 2, 2008, pp. 1-17.

[3] Al-Sayari S.: The Influence of Wettability and Carbon Dioxide Injection on Hydrocarbon Recovery. Imperial College of London, London 2009.

[4] Anderson G.A.: Simulation of Chemical Flood Enhanced Oil Recovery Processes Including the Effects of Reservoir Wettability. The University of Texas at Austin, USA 2006. 
[5] Anderson W.G.: Wettability literature survey-part 1: rock/oil/brine interactions and the effects of core handling on wettability. Journal of Petroleum Technology, vol. 38, no. 11, 1986, pp. 1125-1144.

[6] Anderson W.G.: Wettability Literature Survey. Part 2: Wettability Measurement. Journal of Petroleum Technology, vol. 38, iss.12, 1986, pp. 1246-1262.

[7] Anderson W.G.: Wettability Literature Survey. Part 3: The Effects of Wettability on the Electrical Properties of Porous Media. Journal of Petroleum Technology, vol. 38, iss. 12, 1986, pp. 1371-1378.

[8] Anderson W.G.: Wettability Literature Survey - Part 4: Effects of Wettability on Capillary Pressure. Journal of Petroleum Technology, vol. 39, 1987, pp. 1283-1300.

[9] Anderson W.G.: Wettability Literature Survey. Part 5: The Effects of Wettability on Relative Permeability. SPE, Journal of Petroleum Technology 1987, vol. 39, iss. 11, pp. 1453-1468.

[10] Donaldson E.C., Alam W.: Wettability. Texas 2008.

[11] Donaldson E.C., Siddiqui T.K.: Relationship between the Archie saturation exponent and wettability. SPE $62^{\text {nd }}$ Annual Technical Conference, Dallas 1987, pp. 1-8.

[12] Donaldson E.C., Tiab D.: Petrophysics: Theory and Practice of Measuring Reservoir Rock and Fluid Transport Properties. Gulf Publishing Company, Waltham, USA 1996.

[13] Falode O., Manuel E.: Wettability Effects on Capillary Pressure, Relative Permeability, andIrredcucible Saturation Using Porous Plate. Journal of Petroleum Engineering 2014, vol. 14, pp. 1-12.

[14] Graue A., Bognø T., Moe R.W., Baldwin B.A., Spinler E.A., Maloney D., Tobola D.P.: Impacts of Wettability on Capillary Pressure and Relative Permeability. International Symposium of Core Analysts, Colorado 1999.

[15] Hamon G., Bennes M.: Two-phase flow rock typing: Another approach. Journal of Petrophysics, vol. 45, no. 5, 2004, pp. 433-444.

[16] http://perminc.com/.

[17] Hui M.H., Blunt M.J.: Effects of wettability on three-phase flow in porous media. Journal of Physical Chemistry, vol. 104, no. 16, 2000, pp. 3833-3845.

[18] Keller G.V.: Effect of wettability on the electrical resistivity of sands. Oil Gas Journal, vol. 51, 1953, pp. 62-65.

[19] Killins C.R., Nielsen R.F., Calhoum J.C.: Capillary desaturation and imbibition in porous rocks. Producers Monthly, vol. 18, no. 2, 1953, pp. 30-39.

[20] Kulynych V.: The influence of wettability on oil recovery. AGH Drilling, Oil, Gas, vol. 32, no. 3, 2015, pp. 493-502.

[21] Lake L.W.: Enhanced Oil Recovery. Prentice-Hall, Englewood Cliffs NJ, USA, 1989.

[22] Longeron D.G., Argaud M.J., Bouvier L.: Resistivity Index and capillary Pressure Measurements Under Reservoir Conditions Using Crude Oil. Annual Technical Conference, San Antonio, Texas 1989.

[23] Longeron D.G., Yahya F.A.: Laboratory Measurements of Cementation Factor and Saturation Exponent under Reservoir Conditions on Asab Reservoir Rock Samples. Society of Core Analysts Annual Technical Conference, San Antonio, Texas 1991.

[24] Mahmood S.M., Maerefat N.L., Chang M.M.: Laboratory Measurement of Electrical Resistivity at Reservoir Conditions. Annual Technical Conference, Houston, Texas, 1988. 
[25] Mikhailov N.N., Motorova K.A., Sechina L.S.: Microstructural wettability of oil and gas reservoirs. Międzynarodowa Konferencja Naukowo-Techniczna Geopetrol, Kraków 2016, pp. 149-155.

[26] Morgan W.B., Pirson S.J.: The effect of fractional wettability on the Archie saturation exponent. SPWLA $5^{\text {th }}$ Annual Logging Symposium, Midland, Texas 1964, pp. 1-13.

[27] Morrow N.R.: Capillary pressure correlations for uniformly wetted porous media. Journal of Canadian Petroleum Technology, vol. 15, no. 4, 1976, pp. 49-69.

[28] Morrow N.R., Cram P.J., Mccaffery F.G.: Displacement Studies in Dolomite With Wettability Control by Octanoic Acid. SPE Journal, vol. 13, no. 4, 1973, pp. 221-232.

[29] Morrow N.R., Lim H.T., Ward J.S.: Effect of Crude-Oil-Induced Wettability Changes on Oil Recovery. Journal of SPE Formation Evaluation, vol. 1, no. 1, 1986, pp. 89-103.

[30] Mungan N., Moore E.J.: Certain wettability effects on electrical resistivity in porous media. Journal of Canadian Petroleum Technology, vol. 7, no. 1, 1968, pp. 20-25.

[31] O'Carroll D.M., Abriola L.M., Polityka C.A., Bradford S.A., Demond A. H.: Prediction of two-phase capillary pressure-saturation relationships in fractional wettability systems. Journal of Contaminant Hydrology, vol. 77, no. 4, 2005, pp. 247-270.

[32] Owens W.W., Archer D.L.: Effect of rock wettability on oil-water relative permeability relationships. Journal of Petroleum Technology, vol. 23, no. 7, 1970, pp. 873-878.

[33] Sondena E., Bratteli F., Kolltveit K., Normann H.P.: A Comparison between Capillary Pressure Data and Saturation Exponents obtained at Ambient Conditions and at Reservoir Conditions. 64nd Annual Technical Conference, San Antonio, Texas 1989.

[34] Sondena E., Bratteli F., Kolltveit K., Normann H.P.: The Effect of Reservoir Conditions, and Wettability on Electrical Resistivity. SPE Asia-Pacific Conference, Perth, Australia 1991.

[35] Sweeney S.A., Jennings H.Y.: Effect of wettability on electrical resistivity of carbonate rock from a petroleum reservoir. Journal of Physical Chemistry, vol. 64, no. 5, 1960, pp. 551-553.

[36] Willhite G.P.: Waterflooding. Society of Petroleum Engineers, vol. 3, Richardson, Texas 1986. 